\title{
Long-term contrafreeloading in rats during continuous sessions
}

\author{
SANDRA RUTTER and JOHN A. NEVIN \\ University of New Hampshire, Durham, New Hampshire
}

\begin{abstract}
Two rats lived in experimental chambers with continuous access to free food. Leverpressing was reinforced with food on various ratio schedules. Both rats maintained leverpressing under these conditions for several months; however, leverpressing was not an increasing function of the ratio, as previously found for rats in continuous sessions without alternative food. Instead, the function was nonmonotonic or decreasing, and the proportion of food obtained by leverpressing decreased continuously as the ratio increased. These findings are consistent with an economic analysis of the elasticity of demand.
\end{abstract}

In this study, we explored two apparently unrelated problems: contrafreeloading, defined as the establishment and maintenance of operant behavior by food reinforcement despite the simultaneous availability of free food; and performance on ratio schedules in closed economies.

Contrafreeloading was first demonstrated in rats by Jensen (1963), who studied food-deprived rats in single 40min test sessions and showed that leverpressing on a continuous reinforcement (CRF) schedule in the presence of free food was an increasing function of the number of reinforcers before the free food was introduced. Similar procedures have generally been employed in subsequent research, but it is not necessary for rats to have experience with response-contingent reinforcement or to be tested in discrete sessions with controlled deprivation in order for them to exhibit contrafreeloading. When naive rats were continuously housed in operant chambers with both free and response-contingent food available on CRF, leverpressing was acquired and maintained for 30 or 100 days (Coburn \& Tarte, 1976; Kopp, Bourland, Tarte, \& Vernon, 1976).

When rats live continuously in experimental chambers with no food other than that produced by responding, their daily response rates increase as the value of a fixed ratio (FR) schedule is increased from CRF to FR240, so that roughly constant food intake is maintained (Collier, Hirsch, \& Hamlin, 1972; see also Collier, Johnson, Hill, \& Kaufman, 1986). This situation is referred to as a closed economy (see, e.g., Collier, 1983; Hursh, 1984). The hallmark of a closed economy is that the experimenter does not arrange extraexperimental feeding, so that the subject regulates its own deprivation level. This aspect of a closed economy is maintained even if free food is continuously available within the experimental environment. We therefore examined performance in a closed

Correspondence should be addressed to Sandra Rutter, Department of Psychology, Conant Hall, University of New Hampshire, Durham, NH 03824-3567. economy with free food available, and asked whether rats would adjust their response rates to obtain a constant number of response-contingent food reinforcers when ratio schedules were varied, as they do when there is no other source of food.

\section{METHOD}

\section{Subjects and Apparatus}

Two male Harvard-strain brown rats served. They each lived continuously in a standard Lehigh Valley operant chamber equipped with a lever and a feeder that dispensed 45-mg Noyes pellets. A metal dish with a capacity of $150 \mathrm{~g}$ of laboratory chow was placed in the chamber, and filled with previously weighed chow daily. Water was always available. There was no houselight, but the chamber was not enclosed, so that the rats received continuous dim lighting from an adjacent room, which also housed the electromechanical control equipment.

\section{Procedure}

Before the experiment began, Rat 1 and a second rat (not subsequently tested, due to apparatus failure) were exposed alternately to freely available Purina chow and Noyes pellets for 30 days. Both rats consumed about 1-2 $\mathrm{g}$ per day more chow than pellets.

The experiment began when the rats were about 2.5 months old. They were placed directly on CRF in the presence of free chow, and were then exposed to ascending series of FR and variable ratio (VR) schedules and extinction, with replication of CRF, in the following orders:

Rat 1, VR series: CRF, VR4, 5, 7, 9, 12, 18, 43, Ext.

Rat 1, FR series: CRF, FR2 through 10, 12, 14.

Rat 2, FR series: CRF, FR 2 through 8, Ext, CRF, FR2, 5.

Rat 2, VR series: VR 3, 4, 7, 11, 15, 24, 4 .

Each condition remained in effect for a minimum of 10 days, or continued if necessary until the number of responses per day appeared stable. Conditions lasted an average of 17 days for Rat 1 and 12 days for Rat 2. Thus, the total duration of the experiment was 14 months for Rat 1 and 8.5 months for Rat 2 .

\section{RESULTS}

Both subjects began leverpressing almost immediately after being placed in their chambers, despite the presence of free food and the lack of history of food deprivation or reinforcement. On the average, $34 \%$ of all food was earned by leverpressing on CRF. Figure 1 shows the number of leverpresses per day and the proportion of all food obtained by leverpressing for both rats at all ratio values. 

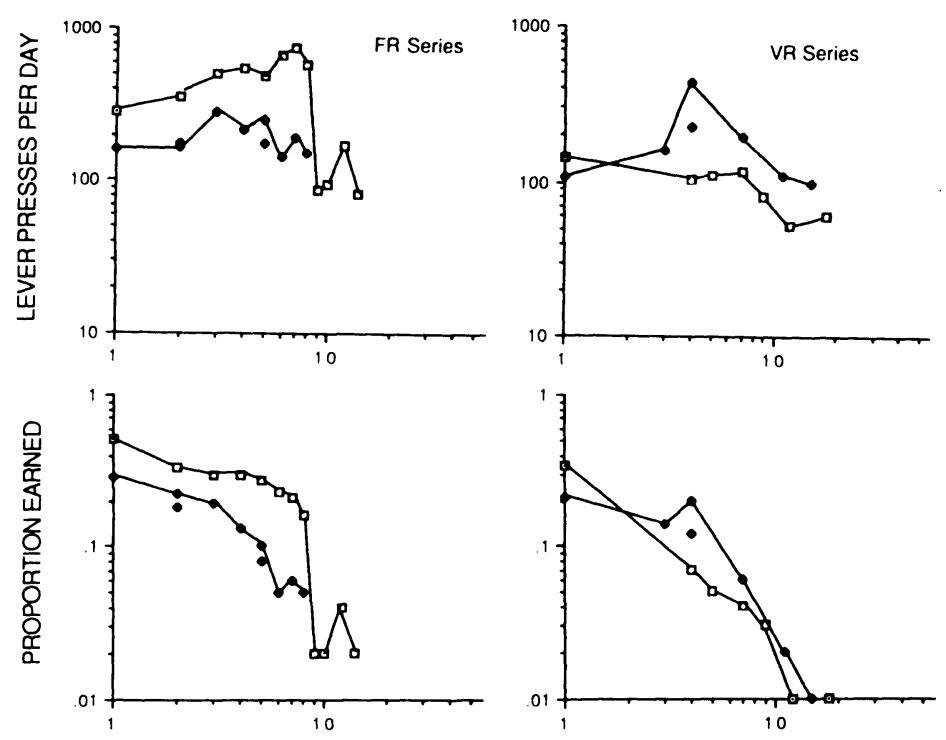

RATIO VALUE

Figure 1. The upper panels show how the number of leverpresses per day depended on the value of the fixed ratio (left panel) or variable ratio (right panel). The lower panels show how the proportion of all food that was earned by leverpressing depended on the ratio values. The data for Rat 1 are shown as unfilled squares, and for Rat 2 as filled diamonds. Unconnected points are replications of previously determined schedule values. Both axes are logarithmic.

The data are medians for the final five sessions at each ratio value. (We omit VR43 and extinction, where leverpressing fell to near zero.)

As the value of the FR schedule was increased, both rats exhibited increases and then decreases in leverpresses per day, with an especially clear maximum for Rat 1 . However, on VR schedules, the maximum was clear only for Rat 2. Except in the case of Rat 1, CRF, replications yielded lower response rates than did initial determinations, but their values were consistent with the overall form of the relation between leverpressing and the ratio requirement. At equivalent schedule values, Rat 1 responded more on FR than on VR schedules, but there was no consistent difference for Rat 2.

There was no systematic relation between the total amount that the rats ate each day and the FR or VR schedule value. Both rats on both schedules exhibited a clear monotonic decrease in the proportion of food obtained by leverpressing; the rate of decrease was somewhat greater on VR than on FR schedules.

\section{DISCUSSION}

Our principal result is that food-reinforced leverpressing in the presence of free food was maintained for many months over a range of ratio schedules and was reestablished after extinction. This result extends those of Coburn and Tarte (1976) and Kopp et al. (1976) on long-term maintenance of contrafreeloading from CRF to ratio schedules, and from 100 days to over a year.
Second, we found that the daily rate of leverpressing on FR schedules was as high as or higher than it was on VR schedules. This result appears to conflict with the finding that VR schedules maintain higher response rates than equivalent FR schedules do (Ferster \& Skinner, 1957). The usual difference between average FR and VR response rates is probably the result of pausing after reinforcement on FR schedules, which could occupy substantial portions of standard experimental sessions. However, in continuous sessions with free food available, so litthe time is allocated to leverpressing that pauses after reinforcement would have negligible effects.

Third, the proportion of daily food intake obtained by leverpressing decreased markedly as the ratio requirement increased. This suggests that demand for pellets earned by leverpressing is more elastic when an alternative supply of food is available than when it is not (see, e.g., Collier et al., 1972, 1986), in keeping with economic accounts of operant behavior (see, e.g., Hursh, 1984). However, the lower plots of Figure 1 suggest that the demand for pellets remains surprisingly inelastic (i.e., slopes are less than -1.0 in double-logarithmic plots) over small fixed ratios. The persistence and replicability of food-reinforced leverpressing on ratio schedules in the presence of free food suggests that this may be an effective testing ground for other economic interpretations of behavior.

\section{REFERENCES}

Coburn, J. F., \& TARTE, R. D. (1976). The effect of rearing environments on the contrafreeloading phenomenon in rats. Journal of the Experimental Analysis of Behavior, 26, 289-294.

ColuIER, G. (1983). Life in a closed economy: The ecology of learning and motivation. In M. D. Zeiler \& P. Harzem (Eds.), Advances in analysis of behaviour: Vol. 3. Biological factors in learning (pp. 223-274). Chichester, England: Wiley.

Collier, G., Hirsch, E., \& Hamlin, P. (1972). The ecological deter- 
minants of reinforcement in the rat. Physiology \& Behavior, 9, 705-716.

Collier, G. H., Johnson, D. F., Hill, W. L., \& Kaufman, L. W. (1986). The economics of the law of effect. Journal of the Experimental Analysis of Behavior, 46, 113-136.

Ferster, C. B., \& SkINNER, B. F. (1957). Schedules of reinforcement. New York: Appleton-Century-Crofts.

Hursh, S. R. (1984). Behavioral economics. Journal of the Experimental Analysis of Behavior, 42, 435-452.
Jensen, G. D. (1963). Preference for bar-pressing over " free-loading" as a function of number of rewarded presses. Journal of Experimental Psychology, 65, 451-454.

Kopp, J., Bourland, G., TARTE, R. D., \& Vernon, C. R. (1976). Acquisition of bar-pressing in non-deprived rats. Psychological Record, 26, $49-54$

(Manuscript received April 28, 1990.)

\section{Announcement}

\section{Human Behavior Bibliography Project}

Readers are invited to participate in a project for the compilation of a Bibliography of Human Behavior.

The project will collect titles from numerous disciplines in which human behavior is researched according to methods of the biological sciences. Some main fields to be covered are Human Evolution, Cultural Evolution (including Prehistory), Human Biology, Behavior Genetics, Ethology, Sociobiology, Biological Anthropology, Social Psychology, Political Science, Experimental Psychology (learning theory), Endocrinology, Brain Sciences, and Psychopathology.

The professional associations sponsoring the project are the Australian Society for Human Biology, the European Sociobiological Society, the Association for Politics and the Life Sciences, and the International Society for Human Ethology.

The bibliography will have a Name and Subject index. Pro-Cite ${ }^{\mathrm{Th}}$ is the project database application. It is expected that the published bibliography will be available in book form and on disk, thus giving users great flexibility.

Because of the bibliography's broad scope, standard methods of title search are being augmented by solicitation of titles. If you choose to submit titles, please use the following format:

- Place your 10 most significant publications at the beginning of the list.

- Write journal titles out in full. For books, please indicate both the publisher's name and place of publication.

- After each title, enter three key words for use in the subject index.

- Titles published after 1980 are preferred. Titles prior to 1980 should be particularly significant.

- Include "in press" publications.

The estimated duration of the project is 2 years. To facilitate inputting, please submit at your earliest convenience.

Submissions should be made to Bibliography Project, Griffith University, Brisbane 4111, Australia (E-Mail humcaton@pegasus.itc.gu.oz.aub). 